\title{
Multielement Determination of Rare Earth Elements in Geochemical Samples by Liquid Chromatography/ Inductively Coupled Plasma Mass Spectrometry
}

\author{
Akihide ItoH*, Tadashi HamanaKa**, Wei Rong**, Katsuya IKEDA**, Hideyuki SAWatari***, \\ Koichi CHIBA** and Hiroki HaRAGUCHI** \\ *Reseach Center for Advanced Waste and Emission Management, Nagoya University, \\ Furo-cho, Chikusa-ku, Nagoya 464-8603, Japan \\ **Department of Applied Chemistry, Graduate School of Engineering, Nagoya University, \\ Furo-cho, Chikusa-ku, Nagoya 464-8603, Japan \\ ***Faculty of Education, Miyagi Institute of Education, Aramaki, Sendai 980-0845, Japan
}

\begin{abstract}
A system of liquid chromatography combined with inductively coupled plasma mass spectrometry (ICP-MS) has been applied to the determination of rare earth elements (REEs) in geochemical standard reference samples. In liquid chromatography, a strong ion-exchange resin was employed as a stationary phase and ammonium lactate was used as a mobile phase. Multielement detection by ICP-MS was performed in the peak hopping mode to measure the chromatograms for all REEs at the corresponding mass numbers. REEs in the standard rock sample (GSJ JB-1) and the standard sediment sample (Pond Sediment; NIES No.2) were determined by the present measurement system without any correction of the matrix effect and polyatomic ion interferences.
\end{abstract}

Keywords Inductively coupled plasma mass spectrometry, rare earth element, geochemical sample, ion exchange chromatography

Recently, inductively coupled plasma mass spectrometry (ICP-MS) has been increasingly used for trace elements in various samples because ICP-MS generally provides much better detection limits by $2-4$ orders of magnitude for most elements. ${ }^{1}$ However, it is noted here that since matrix effects are often serious in ICPMS, ICP-AES is still useful for the determination of major, minor and some trace elements, when the concentrations of these analyte elements are higher than 1 $\mu \mathrm{g} / \mathrm{ml}$ in the analysis solution. In addition, ICP-AES provides better analytical precision than does ICP-MS. Thus, it is desirable to use ICP-MS and ICP-AES, depending on the concentration levels of the analyte elements as well as analytical purposes.

The concentrations and distribution patterns of rare earth elements (REEs), for example in rock samples, generally provide important information about the geochemical evolution and origins of magmatic rocks. Thus, REEs in geological or geochemical samples, such as rocks, sediments and soils, have been determined by neutron activation analysis, inductively coupled plasma atomic emission spectrometry (ICP-AES), and thermal-ionization mass spectrometry. ${ }^{1}$ The present authors previously reported on the determinations of REEs in rock samples ${ }^{2,3}$ and rare earth ores ${ }^{4}$ by con-

T. H. present address: Nippon Denso Co. Ltd., 1-1, Showamachi, Kariya, 448-8661, Japan. ventional ICP-AES with pneumatic nebulization. In addition, we explored the combined LC (liquid chromatography)/ICP-AES system as the analytical method for the determination of REEs in rock samples and REE oxides, where a monochromator system for single-element detection ${ }^{5,6}$ and a polychromator system for simultaneous multielement detection ${ }^{7}$ were employed. In these LC/ICP-AES systems, the main purpose of using LC was to separate the coexisting diverse elements and REEs in rock and other samples so as to eliminate interelement spectral interferences.

Recently, ion chromatography combined with ICPMS was applied to the determination of REEs. ${ }^{8}$ The present authors also reported a LC/ICP-MS system for the chromatographic determination of REEs. ${ }^{9}$ Since ICP-MS generally provides much better detection sensitivities for REEs than does ICP-AES ${ }^{10,11}$, the analytical results for REEs obtained by the LC/ICP-MS system appeared to be more reliable than those by the LC/ICP-AES system. ${ }^{9}, 12$ Thus, in the present experiment the determination of REEs in geological samples has been investigated in more detail by the LC/ICP-MS system. Furthermore, ICP-AES and the LC/ICP-AES system were also used for determining the matrix elements and for investigating the separation efficiencies of the matrix elements from REEs in the sample solutions. 


\section{Experimental}

\section{Instrumentation}

A liquid chromatograph (Model LC-9A, Shimadzu Instrument Co., Kyoto) was used for ion-exchange separation of REEs and concomitant ions with a strong ion-exchange column (TSKgel SP-2SW, TOSOH, Tokyo), where two pumps were used for concentration gradient elution. The sample injection volume into LC was $100 \mu \mathrm{l}$. In ion-exchange separation, ammonium lactate was used as the mobile phase under the concentration gradient program. The experimental conditions for LC operation are given in Table 1.

An ICP-MS instrument (Model SPQ8000, Seiko Instrument Co., Chiba) was used with a concentric nebulizer for pneumatic nebulization. The conventional operating conditions of the ICP instrument are summarized in Table 1. The capillary of the nebulizer in ICPMS was connected through a Teflon tubing to the exit of the LC column to construct a LC/ICP-MS system. Thus, REEs were on-line detected by ICP-MS after ion-exchange separation with LC. In a chromatogram measurement, ICP-MS was operated in the peak hopping mode for multielement detection, where the ICP-MS signals at $15 \mathrm{~m} / \mathrm{z}$ positions were measured during a single mass-range scanning. The dwell time at each $\mathrm{m} / \mathrm{z}$ was $100 \mathrm{~ms}$, and the chromatograms were drawn by plotting the data every $5 \mathrm{~s}$. The total acquisition time for chromatogram measurements was $2400 \mathrm{~s}$, which resulted in observations of each chromatogram with 480 points at each $\mathrm{m} / z$. The thus-measured chromatograms are shown in Figs. 1, 3 and 4. In these chromatograms, the maximum intensity of each peak was normalized to 1 according to the computer program employed in the present experiment, although the

Table 1 Instrumentation and experimental conditions for the LC / ICP-MS system

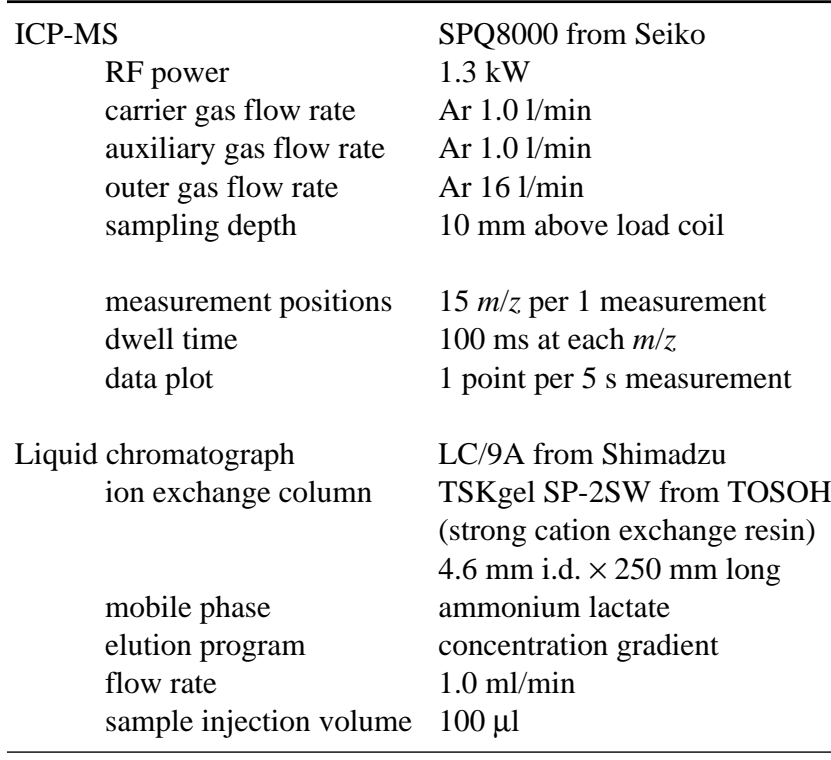

peak intensities were obtained as the real ion counts. In addition, the curves of the chromatograms were smoothed by taking the average of each 5 points.

\section{Chemicals and samples}

The chemicals used were of analytical-reagent grade. The working standard solution of REEs was prepared by mixing the commercially available standard stock solutions of REEs $(1000 \mu \mathrm{g} / \mathrm{ml}$ each $)$ for atomic absorption spectrometry, which were purchased from Wako Chemicals Co. (Osaka). Lactic acid and an aqueous ammonia solution were also purchased from Wako Chemicals Co. The ammonium lactate solutions $(\mathrm{pH}$ 4.2) used for the mobile phase were prepared by mixing lactic acid and ammonia solutions. The rock and sediment standard reference samples, issued by the Geological Survey of Japan (Tsukuba) and the National Institute for Environmental Studies (Tsukuba), respectively, were used for analysis. The samples were digested by alkali fusion using $\mathrm{LiBO}_{2}(>99 \%)$ from $\mathrm{E}$. MERCK (Darmstadt, Germany), and finally dissolved with $0.1 \mathrm{M} \mathrm{HNO}_{3}$. The digested samples were subjected to analysis without any further pretreatment.

\section{Results and Discussion}

\section{Chromatographic separation of REEs}

The chromatograms for REEs $(20 \mathrm{ng} / \mathrm{ml}$ each in 0.1 $\mathrm{M} \mathrm{HNO}_{3}$ ) in the mixed standard solution were measured by the present LC/ICP-MS system, and are shown in Fig. 1. In this chromatographic measurement, a strong ion-exchange column of TSKgel SP-2SW was used for separating REEs, and the elution was performed using an ammonium lactate solution as the mobile phase under the following concentration gradient program. In the concentration gradient program, two solutions (A and $\mathbf{B}$ ) with different concentrations of ammonium lactate were used as the mobile phase; the $\mathbf{A}$ and $\mathbf{B}$ solutions were $0.1 \mathrm{M}$ and $0.4 \mathrm{M}$ of ammonium lactate aqueous solution $(\mathrm{pH} 4.2)$, respectively. The gradient program was operated as follows: a $100 \%$ A solution was gradually decreased down to $90 \%$ and B was alternately increased up to $10 \%$ for the first 15 min, the mixture of $90 \% \mathbf{A}$ solution and $10 \% \mathbf{B}$ from $15 \mathrm{~min}$ to $25 \mathrm{~min}$, and then $\mathbf{B}$ solution gradually up to $100 \%$ from $25 \mathrm{~min}$, and finally to $40 \mathrm{~min}$.

The retention times of REEs obtained from the chromatograms shown in Fig. 1 are summarized in Table 2, together with those obtained using $\alpha$-HIBA $(2$ hydroxy-2-methylpropanoic acid) as the mobile phase, which was examined in a previous study. ${ }^{9}$ It can be seen in Fig. 1 that the peak-profile overlappings are observed in the cases of Lu-Yb-Tm-Er and Eu-Sm. In such cases, the differences in the retention times were smaller than $100 \mathrm{~s}$, as is seen in Table 2. However, the present chromatograms for REEs measured in the peak hopping mode were independently observed at each $\mathrm{m} / \mathrm{z}$ under the mass-separated conditions. Thus, the 


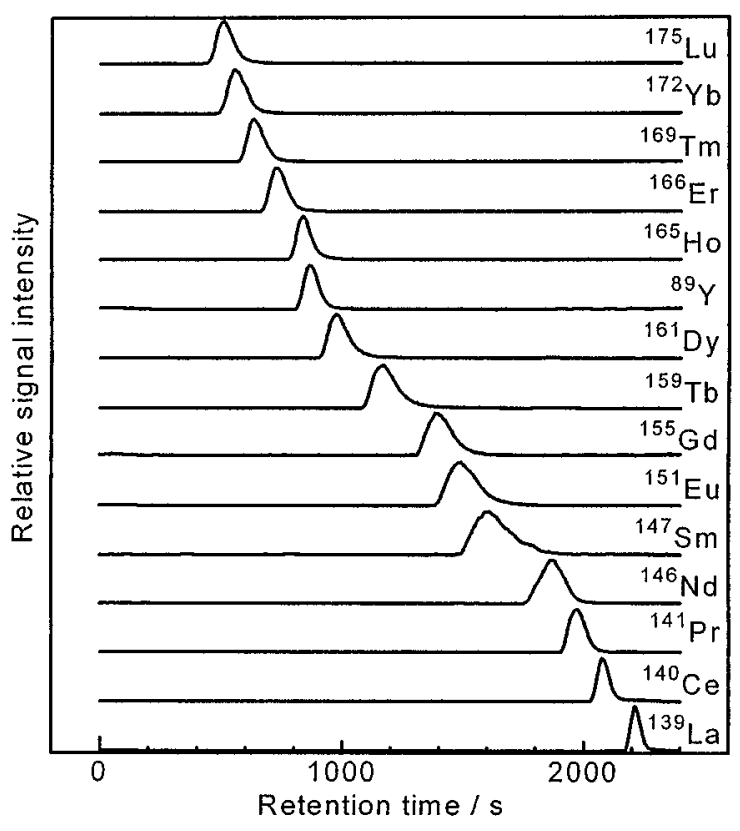

Fig. 1 Chromatograms of rare earth elements in a mixed standard solution with multielement detection of ICP-MS. LC elution, concentration gradient (see text); mobile phase, ammonium lactate; separation column, cation exchange resin; flow rate, $1 \mathrm{ml} / \mathrm{min}$; injection volume, $100 \mu \mathrm{l}$.

Table 2 Retention times of rare earth elements obtained by the present LC/ICP-MS system

\begin{tabular}{ccc}
\hline & \multicolumn{2}{c}{ Retention time/s } \\
\cline { 2 - 3 } Element & Ammonium lactate & $\alpha-$ HIBA $^{\mathrm{a}}$ \\
\hline $\mathrm{Lu}$ & 510 & 465 \\
$\mathrm{Yb}$ & 540 & 490 \\
$\mathrm{Tm}$ & 595 & 530 \\
$\mathrm{Er}$ & 685 & 600 \\
$\mathrm{Ho}$ & 840 & 705 \\
$\mathrm{Y}$ & 890 & 770 \\
$\mathrm{Dy}$ & 965 & 870 \\
$\mathrm{~Tb}$ & 1155 & 1130 \\
$\mathrm{Gd}$ & 1340 & 1420 \\
$\mathrm{Eu}$ & 1400 & 1515 \\
$\mathrm{Sm}$ & 1485 & 1615 \\
$\mathrm{Nd}$ & 1685 & 1785 \\
$\mathrm{Pr}$ & 1905 & 1850 \\
$\mathrm{Ce}$ & 2010 & 1915 \\
$\mathrm{La}$ & 2180 & 2070 \\
\hline
\end{tabular}

a. Cited from ref. 9.

overlappings of the peak profiles were not seriously prone to mutual interferences in the determination of REEs by LC/ICP-MS.

In comparison with the results obtained by using ammonium lactate and $\alpha$-HIBA as the mobile phase, the better peak resolution of the chromatographic peaks for REEs were obtained rather in the case of $\alpha$-HIBA. However, better analytical results, especially in terms
Table 3 Detection limits obtained by the LC/ICP-MS system

\begin{tabular}{crcc}
\hline & & \multicolumn{2}{c}{ Detection limit $^{\mathrm{a} / \mathrm{ng} \mathrm{m}^{-1}}$} \\
\cline { 3 - 4 } Element & $m / z$ & LC/ICP-MS $^{\mathrm{b}}$ & Conventional ICP-MS $^{-}$ \\
& & 0.49 & 0.0027 \\
$\mathrm{Y}$ & 89 & 0.23 & 0.006 \\
$\mathrm{La}$ & 139 & 0.13 & 0.0056 \\
$\mathrm{Ce}$ & 140 & 0.044 & 0.0041 \\
$\mathrm{Pr}$ & 141 & 0.051 & 0.0050 \\
$\mathrm{Nd}$ & 146 & 0.054 & 0.0029 \\
$\mathrm{Sm}$ & 147 & 0.055 & 0.0024 \\
$\mathrm{Eu}$ & 151 & 0.032 & 0.0043 \\
$\mathrm{Gd}$ & 155 & 0.063 & 0.0036 \\
$\mathrm{~Tb}$ & 159 & 0.027 & 0.0028 \\
$\mathrm{Dy}$ & 161 & 0.010 & 0.0027 \\
$\mathrm{Ho}$ & 165 & 0.018 & 0.0065 \\
$\mathrm{Er}$ & 166 & 0.010 & 0.0022 \\
$\mathrm{Tm}$ & 169 & 0.022 & 0.0030 \\
$\mathrm{Yb}$ & 172 & 0.027 & 0.0030 \\
$\mathrm{Lu}$ & 175 &
\end{tabular}

a. Detection limits for the standard solution of REEs with direct nebulization.

b. The concentration gradient elution was employed, as described in the text.

of precision, were obtained in the case of ammonium lactate. This may be because the slow elution (longer retention time) of REEs was achieved in the case of ammonium lactate, which allowed a more precise chromatogram measurement in the peak-hopping mode.

\section{Detection limits of REEs obtained by LC/ICP-MS}

The detection limits obtained by the present LC/ICPMS system are summarized in Table 3 together with those obtained by conventional ICP-MS with pneumatic nebulization. The detection limit in LC/ICP-MS was estimated as the concentration corresponding to 3-times the standard deviation $(\sigma)$, which was calculated from the blank intensity measured at each $\mathrm{m} / \mathrm{z}$ of REEs with flowing only the mobile phase $(0.1 \mathrm{M}$ ammonium lactate solution). The present detection limits were poorer by 10 - 180 times for Y, La, Ce, Pr, Nd, Sm, Eu, and Tb compared to those obtained by conventional ICP-MS, while they were less than 10 times for other REEs. It is noted that the detection limits in LC/ICP-MS were poorer for light REEs than those for heavy REEs. Even so, LC/ICP-MS was applicable to the determination of REEs because the natural abundances of light REEs are significantly higher than those of heavy REEs. The present LC/ICP-MS system, thus, provided the following advantages over the conventional ICP-MS, even though the detection limits were fairly deteriorated. First, spectral interferences due to polyatomic ions could be eliminated because of mutual separation of REEs as well as major elements. Second, such mutual separation also helped to reduce matrix effects due to major elements. Thus, it was not necessary to make any internal-standard correction in the present experi- 
Table 4 Concentration of major elements in standard rock sample (GSJ JB-1) and sediment sample (NIES No. 2) determined by ICP-AES

\begin{tabular}{cccccc}
\hline \multirow{2}{*}{ Element } & \multicolumn{2}{c}{ Rock sample } & & \multicolumn{2}{c}{ Sediment sample } \\
\cline { 2 - 3 } \cline { 5 - 6 } & Observed, \% & Certified, \% & & Observed, \% & Certified, \% \\
\hline $\mathrm{Na}$ & 2.04 & 2.05 & & 0.57 & 0.57 \\
$\mathrm{Mg}$ & 4.52 & 4.66 & & 0.85 & 0.81 \\
$\mathrm{Al}$ & 7.07 & 7.69 & & 10.5 & 10.6 \\
$\mathrm{Si}$ & 24.2 & 24.48 & & 22.0 & 22.0 \\
$\mathrm{~K}$ & 1.09 & 1.19 & & 0.52 & 0.68 \\
$\mathrm{Ca}$ & 6.64 & 6.61 & & 0.80 & 0.81 \\
$\mathrm{Ti}$ & 0.79 & 0.80 & & 0.60 & 0.64 \\
$\mathrm{Fe}$ & 6.17 & 6.29 & & 6.8 & 6.53 \\
Total & 53.03 & 53.77 & & 42.64 & 42.64 \\
\hline
\end{tabular}

mental system.

\section{Chromatographic retention behaviors of major elements}

The concentrations of major elements in the rock and sediment samples examined here were determined by ICP-AES (inductively coupled plasma atomic emission spectrometry), where the ICP-AES instrument (Model Plasma AtomComp MK II from Jarrell-Ash, Franklin, MA, USA) was operated under the usual experimental conditions. ${ }^{7}$ Before the analysis, the rock and sediment samples were digested, as mentioned later. The analytical results are summarized in Table 4, together with the certified values. As can be seen in Table 4, the concentrations of the alkali and alkaline earth elements $(\mathrm{Na}$, $\mathrm{K}, \mathrm{Mg}$, and $\mathrm{Ca}$ ) in the rock sample were much higher than those in the sediment sample. In general, a large amount of major constituents in the samples often causes matrix effects in the ICP-MS measurement. Thus, the ion-exchange separation of the major elements in the rock sample was further examined using the LC/ICP-AES system.

In a chromatogram measurement by LC/ICP-AES, the same ion-exchange column and experimental conditions as those shown in Fig. 1 were employed. The LC/ICP-AES measurement system, which was developed in the present laboratory, was used in the experiment in similar manner to the previous experiment. ${ }^{7}$ The test solution for the chromatogram measurements was prepared so as to match the concentrations of the major elements with those in the digested rock sample solution, in which $\mathrm{Li}$ was also added, because $\mathrm{LiBO}_{2}$ was used for fusion of the samples.

The ion-exchange chromatograms of the major elements are shown in Fig. 2. Compared to the chromatograms in Fig. 1, it can be seen from Fig. 2 that the retention times of $\mathrm{Ca}, \mathrm{Si}, \mathrm{Ti}$, and $\mathrm{Fe}$ were shorter than those of REEs, and those of $\mathrm{K}$ and $\mathrm{Mg}$ were much longer. Thus, these elements may not interfere with REEs in the chromatographic measurement by LC/ICPMS. However, $\mathrm{Li}, \mathrm{Na}$, and $\mathrm{Al}$ eluted in the retention

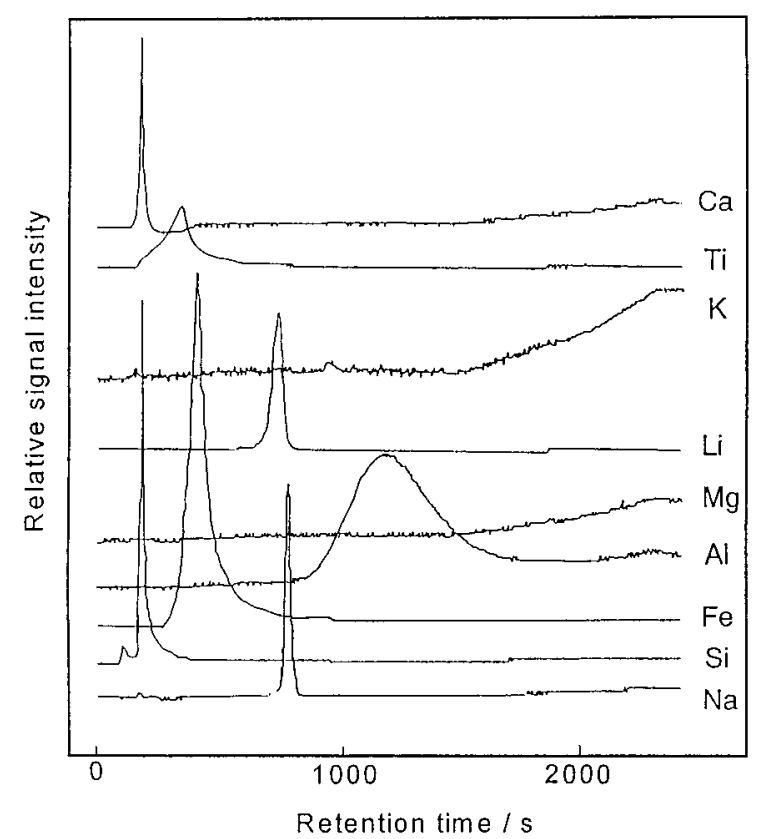

Fig. 2 Chromatograms of the major constituents in the standard rock sample (JB-1) with multielement detection by ICPAES. Chromatographic experimental conditions, same as in Fig. 1. Concentrations of major concentrations in the test solution of rock sample (JB-1): Li $4.17 \mu \mathrm{g} / \mathrm{ml}$, Na $39.6 \mu \mathrm{g} / \mathrm{ml}$, Mg $87.7 \mu \mathrm{g} / \mathrm{ml}$, Si $137 \mu \mathrm{g} / \mathrm{ml}, \mathrm{K} 21.1 \mu \mathrm{g} / \mathrm{ml}$, Ca $128 \mu \mathrm{g} / \mathrm{ml}$, $\mathrm{Fe} 120 \mu \mathrm{g} / \mathrm{ml}$.

time range of REEs. The peak-profile overlappings are observed for the cases of Li-Er, Na-Y, and Al-Tb. Thus, the matrix effects of these elements should be taken into consideration in the LC/ICP-MS measurement.

\section{Determination of REEs in standard rock and sediment samples}

The standard rock sample (JB-1: basalt) issued from the Geological Survey of Japan was analyzed by the present LC/ICP-MS system. The rock sample ( $c a .0 .2$ g) was digested by alkali fusion using $\mathrm{LiBO}_{2}(c a .0 .3 \mathrm{~g}$ ) in a platinum crucible, which was heated on a burner for $15 \mathrm{~min}$. After cooling, the residue was dissolved in $100 \mathrm{ml}$ of $0.1 \mathrm{M} \mathrm{HNO}_{3}$. The rock sample solution was subjected to analysis by the LC/ICP-MS system without any further pretreatment. Element-selective chromatograms of REEs in the rock sample are shown in Fig. 3. In the determination procedure, the standard solution of REEs was first injected into LC for standardization, and then the rock-sample solution was injected for analysis. REEs were determined by the peak-area measurement method. The analytical results are summarized in Table 5, along with the certified values and relative standard deviation. As can be seen in Table 5, the observed values in the present method are almost in good agreement with the certified values ${ }^{13}$, although the values for light REEs (Y - Sm) were rather smaller than the certified values.

REEs in the pond-sediment reference material (NIES 


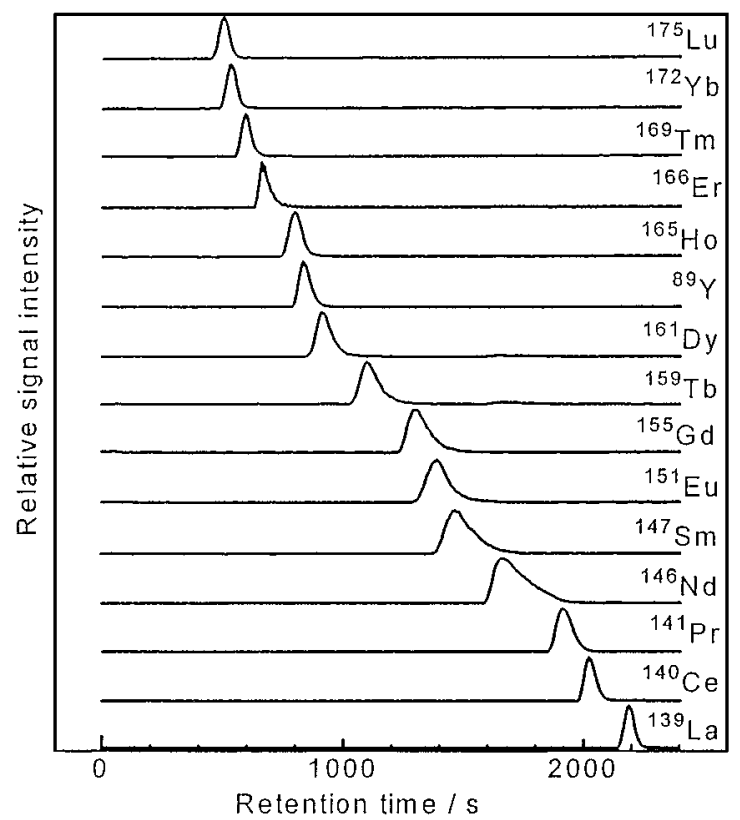

Fig. 3 Chromatograms of rare earth elements in the standard rock sample (JB-1) with multielement detection of ICP-MS. Experimental conditions, same as in Fig. 1.

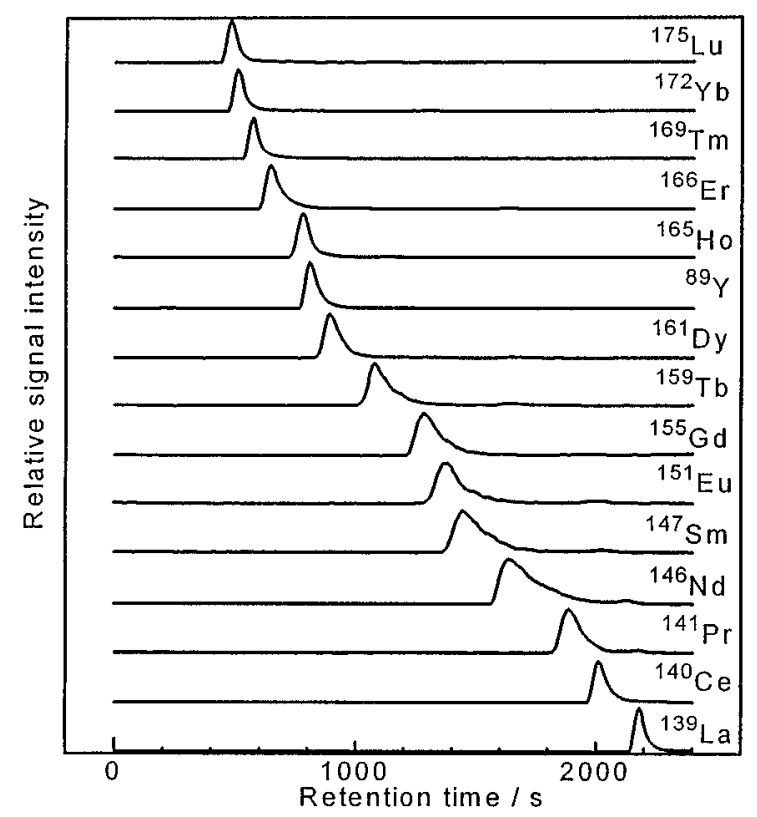

Fig. 4 Chromatograms of rare earth elements in the standard sediment sample (NIES No. 2) with multielement detection of ICP-MS. Experimental conditions, same as in Fig. 1.

Table 5 Analytical results for the determination of rare earth elements in the standard rock sample (GSJ JB-1) and sediment sample (NIES No. 2) obtained by the LC/ICP-MS system

\begin{tabular}{|c|c|c|c|c|c|c|}
\hline \multirow[b]{2}{*}{ Element } & \multicolumn{3}{|c|}{ Rock sample } & \multicolumn{3}{|c|}{ Sediment sample } \\
\hline & $\begin{array}{l}\text { Observed/ } \\
\mu \mathrm{g} \mathrm{g}^{-1}\end{array}$ & $\mathrm{RSD}^{\mathrm{a}}$ & $\begin{array}{c}\text { Certified }{ }^{\mathrm{b}} / \\
\mu \mathrm{g} \mathrm{g}^{-1}\end{array}$ & $\begin{array}{c}\text { Observed/ } \\
\mu \mathrm{g} \mathrm{g}^{-1}\end{array}$ & $\mathrm{RSD}^{\mathrm{a}}$ & $\begin{array}{c}\text { Certified }{ }^{\mathrm{c} /} \\
\mu \mathrm{g} \mathrm{g}^{-1}\end{array}$ \\
\hline $\mathrm{Y}$ & 18.9 & 0.076 & 24.4 & 23.5 & 0.068 & 23.2 \\
\hline $\mathrm{La}$ & 33.6 & 0.031 & 37.9 & 15.7 & 0.088 & 16.7 \\
\hline $\mathrm{Ce}$ & 56.2 & 0.013 & 66.7 & 31.8 & 0.042 & 36.1 \\
\hline $\operatorname{Pr}$ & 6.5 & 0.036 & 7.0 & 4.1 & 0.037 & 4.2 \\
\hline $\mathrm{Nd}$ & 22.8 & 0.029 & 26.7 & 16.4 & 0.033 & 18.4 \\
\hline $\mathrm{Sm}$ & 4.6 & 0.029 & 5.1 & 3.8 & 0.026 & 4.1 \\
\hline $\mathrm{Eu}$ & 1.4 & 0.061 & 1.5 & 1.1 & 0.038 & 1.2 \\
\hline $\mathrm{Gd}$ & 4.4 & 0.039 & 4.9 & 4.2 & 0.019 & 4.6 \\
\hline $\mathrm{Tb}$ & 0.80 & 0.16 & 0.84 & 0.6 & 0.039 & 0.7 \\
\hline Dy & 3.7 & 0.022 & 4.1 & 4.1 & 0.041 & 4.6 \\
\hline Ho & 0.7 & 0.047 & 0.8 & 0.8 & 0.11 & 0.9 \\
\hline $\mathrm{Er}$ & 2.3 & 0.057 & 2.3 & 2.5 & 0.056 & 2.7 \\
\hline $\mathrm{Tm}$ & 0.30 & 0.072 & 0.35 & 0.4 & 0.034 & 0.4 \\
\hline $\mathrm{Yb}$ & 2.1 & 0.033 & 2.2 & 2.5 & 0.097 & 2.6 \\
\hline $\mathrm{Lu}$ & 0.32 & 0.096 & 0.31 & 0.4 & 0.038 & 0.4 \\
\hline
\end{tabular}

a. Relative standard deviation, which was estimated from the 3-times measurements. b. Cited from ref. 13. c. Cited from ref. 14.

No. 2) issued from the National Institute for Environmental Studies of Japan, which was collected from a pond in the campus of the University of Tokyo, located in the central part of Tokyo City, were also determined by the LC/ICP-MS system. The sediment sample was digested in a similar manner to the rock sample using $\mathrm{LiBO}_{2}$. Chromatograms of REEs for the sediment sample are shown in Fig. 4. The determination of REEs was also performed by the peak-area measurement method. The analytical results are also sum- marized in Table 5, along with the certified values. As can be seen in Table 5, the observed values for all REEs in the sediment sample are in good agreement with the certified values. ${ }^{14}$

It is noted here that the concentrations of REEs in the rock and sediment samples are almost at the same level for each other, though rather smaller values were obtained for light REEs in the rock sample examined in the present experiment, as mentioned above. The deviation in the analytical values for light REEs in the rock 
sample may be partly due to the poorer detection limits for light REEs than those for heavy REEs, obtained in the present LC/ICP-MS system. Another reason is possibly ascribed to some matrix effects caused by the major constituents. ${ }^{6}$ It is noted here that the concentrations of alkali and alkaline earth elements $(\mathrm{Na}, \mathrm{K}, \mathrm{Mg}$ and $\mathrm{Ca}$ ) in the rock sample were much higher than those in the sediment sample. It can be seen in Table 5 that the observed value of $\mathrm{Y}$ in the rock sample was significantly smaller than its certified value. As can be seen in Fig. 2, the retention time of $\mathrm{Na}$ was almost consistent with that of Y. Thus, it is considered that the coexistence of a large amount of $\mathrm{Na}$ in the rock sample possibly resulted in a smaller value of $\mathrm{Y}$ determined by LC/ICP-MS. It was reported in a previous paper ${ }^{15}$ that a large amount of coexisting $\mathrm{Na}$ suppressed the ion counts of alkaline-earth elements in the ICP-MS measurement. This result was interpreted by suppression of ionization due to easily ionizable elements (EIEs). The $\mathrm{Na}$ concentration of the rock sample was larger by about 4 times than that of the sediment sample. Therefore, the smaller observed value of $\mathrm{Y}$ in the rock sample might be caused by ionization suppression due to $\mathrm{Na}$, which co-eluted at almost the same retention time as $\mathrm{Y}$ in the LC separation. Although no correction was made in terms of matrix effects due to $\mathrm{Na}$ in the present experiment, matrix matching of the working standard solution with the sample solution may be recommended to obtain more accurate analytical values, even in the LC/ICP-MS measurement, when the concentrations of the major constituents, especially in the case of easily ionizable elements, are too high. Since the ionization energies of $\mathrm{Li}$ and $\mathrm{Al}$ are larger than that of $\mathrm{Na}$, the matrix effects due to $\mathrm{Li}$ and $\mathrm{Al}$ were perhaps negligibly small in the present case.

The system of liquid chromatography combined with ICP-MS has been applied to the determination of rare earth elements (REEs) in geochemical reference materials: rock sample (GSJ JB-1) and sediment sample (Pond Sediment; NIES No.2). As a result, REEs in these samples could be determined by the present measurement system without any correction of the matrix effect and polyatomic ion interferences, although possi- ble ionization interfernce of Na with $\mathrm{Y}$ and light REEs in the rock sample was observed. Thus, the present method is applicable to the determination of REEs in geological samples decomposed by alkali fusion, because sample pretreatment is minimized with the aid of precolumn separation of the analytes from concomitant elements.

\section{References}

1. C. Vandecasteele and C. B. Block, "Modern Methods for Trace Element Determination", John Wiley \& Sons, New York, Chichester, Brisbane, Tronto, 1993.

2. K. Toyoda and H. Haraguchi, Chem. Lett., 1985, 981.

3. K. Toyoda and H. Haraguchi, Bull. Chem. Soc. Jpn., 60, 933 (1987).

4. K. Iwasaki, K. Fuwa and H. Haraguchi, Anal. Chim. Acta, 183, 239 (1986).

5. K. Yoshida, K. Fuwa and H. Haraguchi, Chem. Lett., 1983, 1879.

6. K. Yoshida and H. Haraguchi, Anal. Chem., 56, 2580 (1984).

7. H. Sawatari, T. Asano, X. Hu, A. Itoh and H. Haraguchi, Bull. Chem. Soc. Jpn., 68, 898 (1995).

8. K. Kawabata, Y. Kishi, O. Kawaguchi, Y. Watanabe and Y. Inoue, Anal. Chem., 63, 2137 (1991).

9. T. Hamanaka, A. Itoh, S. Itoh, H. Sawatari and H. Haraguchi, Chem. Lett., 1995, 363.

10. A. Montaser and D. W. Golightly, "Inductively Coupled Plasmas in Analytical Atomic Spectrometly", VCH Publisher, New York, 1992.

11. K. E. Jarris, A. L. Gray and R. S. Houk, "Handbook of Inductively Coupled Plasma Mass Spectrometry”, Blackie Academic Professional, London, 1992.

12. A. Itoh, C. Kimata, H. Miwa, H. Sawatari and H. Haraguchi, Bull. Chem. Soc. Jpn., 69, 3469 (1996).

13. S. Terashima, N. Imai, S. Itoh and A. Ando, Geochemical J., 29, 91 (1995).

14. K. Okamoto, Research Report from the National Institute for Environmental Studies, 38, 1 (1982).

15. H. Fujimori, H. Sawatari, K. Chiba and H. Haraguchi, Anal. Sci., 12, 465 (1995).

(Received June 19, 1998)

(Accepted September 24, 1998) 